\title{
The relationship between acquired impairments of executive function and behaviour change in adults with Down syndrome.
}

\section{Adams, D., \& Oliver, C. (2011)}

Published in: Journal of Intellectual Disability Research, 54: 393-405.

$$
\text { doi: 10.1111/j.1365-2788.2010.01271.x }
$$

Keywords: Down syndrome, dementia, Alzheimer's disease, executive function, aging, neuropsychological assessment, 


\begin{abstract}
Background

The latter stages of dementia in individuals with Down syndrome are well documented, however, earlier cognitive and behavioural changes have only recently been described. Holland et al. (2000) suggested such earlysigns of dementia in this population are behavioural and are similar to those seen in frontotemporal dementia, but there is, as yet, no evidence to determine whether such behavioural changes are associated with a declines in specific cognitive functions, including those associated with the frontal lobes.
\end{abstract}

\title{
Method
}

A longitudinal design of three time points across 16 months was employed across 30 adults with Down syndrome aged 30 and over. Measures of cognition (Neuropsychological Assessment of Dementia in Individuals with Intellectual Disabilities; NAID), receptive language (British Picture Vocabulary Scales; BPVS), adaptive behaviour (Vineland Adaptive Behavior Scales; VABS), behavioural excesses and behavioural deficits (Assessment for Adults with Developmental Disabilities; AADS) and measures tests of executive functioning were completed at each time point. Using a data driven method, cognitive deterioration was determined using the Reliable Change Index on performance on the NAID across the duration of the study. Performance on the remaining measures were then compared between those with $(n=10)$ and those without $(n=20)$ cognitive deterioration.

\section{Results}

Only individuals with cognitive deterioration showed decreases on measures of executive function and significant ichanges in behaviour across the duration of the study, which were not solely due to declines in memory. There were no changes between the groups on levels of adaptive behaviour.

\section{Conclusion}

Even in the early stages of cognitive deterioration, specific behavioural changes can be identified that are not present in those without cognitive deterioration. The differing effects of cognitive deterioration on behavioural excesses and deficits are discussed in relation to potentially differing underlying neuropathological causes. 
Introduction

The prevalence of Alzheimer related dementia in adults with Down syndrome ranges from 0 to 2\% in those aged under 40 to over $40 \%$ in those above the age of 60 (Prasher \& Filer, 1995; Holland, Hon, Huppert, Stevens, \& Watson, 1998, Holland, Hon, Huppert, \& Stevens, 2000). The latter stages of dementia in individuals with Down syndrome are well documented and are similar to those seen in the general population with regard to clinical and neuropathological phenomena (e.g. Oliver, Crayton, Holland, Hall, \& Bradbury, 1998; Nelson, Orme, Osann, \& Lott, 2001; Prasher et $a l ., 2003)$. Cognitive and behavioural changes prior to diagnosis or in the early stages of dementia have only been described recently. Longitudinal studies have shown decline in working memory (akin to that noted within Mild Cognitive Impairment in the general population) to be an early indicator of dementia in individuals with Down syndrome (Devenny, Zimmerli, Kittler and Krinsky-McHale, 2002; Oliver et al., 1998; Nelson et al, 2001). This may be apparent even in those aged over 40 who do not evidence signs of other cognitive impairments (In review citation withheld for blind review, Oliver, Holland, Hall \& Crayton. 2005).

Early studies of potential difference in the clinical presentation of the course for dementia in Down syndrome relied on informant reports. For example, Holland et al. (1998; 2000) compared those with and without a clinical diagnosis of dementia of the Alzheimer's type on informant based retrospective reports of clinical presentation. More recent studies have adopted a quasi-experimental approach with results suggesting that changes in personality and behaviour, similar to those seen in frontotemporal dementia, are apparent before changes in working memory.or other domains (Ball, Holland, Treppner, Watson, \& Huppert,, 2008; Holland et al., 1998; 2000, Nelson, Lott, Touchette, Satz, \& D'Elia, 1995; Nelson et al., 2001). The conclusions of these studies have been supported by the results of neuropsychological and diagnostic assessments that have confirmed executive function deficits and features of frontotemporal dementia as a component of the presentation of dementia in adults with Down syndrome (Ball et al., 2008). The nature of the potential difference in this aspect of the course of dementia clearly warrants further attention. In addition to these observations it is clear that both behavioural deficits (e.g. skill loss) and behavioural excesses (e.g. wandering) are evident in the course of dementia with the latter more likely to lead to referral (Adams et al., 2008; Urv, Zigman, \& Silverman, 2003).

Holland et al. (2000) hypothesise that these behavioural and 'personality' changes noted in the early stages of dementia ("frontal type dementia") in adults with Down syndrome can be understood by reference to the "reserve capacity" hypothesis (Mortimer, 1988). Briefly, this model suggests that, 
as development of the frontal lobes is compromised in Down syndrome (see Pinter, Eliez, Schmitt, Capone \& Reiss, 2001), the early neuropathological changes associated with Alzheimer's disease have a comparatively disproportionate effect on cognitive functions associated with the frontal lobes and thus a clinical presentation similar to that of frontal variant frontotemporal dementia. The consistent clinical observation of "dysexecutive" behaviour and cognition arising from damage to the frontal lobes has resulted in executive dysfunction and frontal lobe dysfunction becoming strongly associated. Whilst many (e.g. Baddeley, 1998) argue that many individuals exhibiting "frontal like" symptoms have no impairment to their frontal lobes and many individuals with frontal lobe damage show no impairment on tests of executive function, there is a wealth of evidence suggesting that the frontal lobes and executive function are at least strongly, if not essentially, linked (Stuss \& Benson, 1984).

There is therefore, good reason to pursue the assessment of executive dysfunction when documenting early decline in the course of dementia in adults with Down syndrome. Specifically, objective neuropsychological evidence of decline in abilities related to the frontal lobes (such as those challenged in tests of executive function) in adults with Down syndrome showing cognitive deterioration would strengthen Holland et al.'s (2000) assertion that the compromised development of the frontal lobes interacts with emerging neuropathology early in the course of dementia. The relationship between impaired executive function and emergent behavioural change can be evaluated in a prospective study to elucidate the relationship between cognitive deficits and behavioural excess and deficits.

In this study we employ a longitudinal design to examine the relationship between impairments in working memory, executive function and behaviour in adults with Down syndrome. We hypothesise that: 1) adults with Down syndrome showing cognitive deterioration will show greater impairment on measures of executive function in comparison to those without cognitive deterioration 2) cognitive deterioration will be associated with behavioural excesses and behavioural deficits and 3) behavioural excesses will be associated with executive dysfunction. In addition to testing these hypotheses we also evaluate the impact of behavioural deficits and excesses on carers and the person themself.

\section{Methods}

$\underline{\text { Participants }}$ 
Participants were drawn from a sample of 39 adults with Down syndrome (diagnosis of which was confirmed either clinically or by karyotyping) who had participated in a longitudinal study of ageing in adults with Down syndrome (see Adams et al., 2008). To appraise change over time, individuals showing floor effects on the neuropsychological assessment battery at baseline assessment were excluded. The remaining sample $(n=30)$ consisted of $15(50 \%)$ males and 15 (50\%) females with Down syndrome aged between 34 and 64 years (mean=44.5, sd=7.5). Nine participants lived with their family (27.3\%), 21 lived in residential homes (72.7\%) and almost all $(n=29)$ participants attended a day placement (96.7\%). Using carer reports, no participants were registered blind or deaf and all participants were able to speak at least single words. Based upon clinical interviews and informant reports conducted at baseline, 9 (30\%) participants from the total sample had been diagnosed with hypothyroidism and were currently prescribed corrective medication.

\section{$\underline{\text { Measures }}$}

An extensive battery of direct and informant based measures were completed at baseline assessment and repeated at eight and sixteen month follow ups. These were chosen to cover the main areas known to decline or change in individuals with Down syndrome as they develop dementia; behaviour (see Holland et al., 1998;2000), adaptive behaviour (see Prasher \& Filer, 1995) and executive function (Ball et al., 2008). The British Picture Vocabulary Scales (BPVS; ; Dunn, Dunn, Whetton, \& Burley, 1998) were administered to all participants in order to obtain an estimate of level of functioning that was comparatively resistant to early declines associated with dementia (see Blair, Marczinski, Davis-Faroque, \& Kertesz, 2007). All informant based measures were completed by a family member or care worker who had known the participant for over one year.

\section{Neuropsychological Assessment of Dementia in Individuals with Intellectual Disabilities}

(NAID; formerly known as the Crayton and Oliver test battery, Oliver \& Crayton, 1993). This neuropsychological test battery assesses working memory, agnosia, aphasia and apraxia. It has been described and used in previous studies to delineate the course of decline in adults with Down syndrome (e.g. Crayton, Oliver, Holland, Bradbury, \& Hall, 1998; Oliver et al., 1998) and demonstrates good reliability and concurrent validity, with split-half reliability at subscale level ranging from .82 to .96 and internal consistency (Cronbach's alpha) ranging from .74 to 95 (In review, citation withheld for blind review). The battery comprises seven subscales, the six used within this study were picture naming (maximum score 14), picture identification (maximum score 14), action on request (maximum score 10), picture memory (maximum score 10), object memory (maximum score 10) and memory for sentences (maximum score 39). The NAID can be divided 
into two domains; "early" signs (memory for objects, memory for pictures and memory for sentences) and "late" signs (picture naming, picture identification and action on request) (In review citation withheld for blind review).

Oliver and Adams (2007) suggest the use of the Reliable Change Index statistic (Jacobson et al., 1984; later modified by Christensen \& Mendoza, 1986) to assess if change in an individual's performance on neuropsychological assessments represents a reliable decline (i.e. reflects more than the fluctuations of the measuring instrument). Dependent upon degree of intellectual disability (assessed using the British Picture Vocabulary Scale; Dunn et al., 1998), Oliver and Adams suggest that changes in scores on the NAID can be classified using the reliable change index into a reliable change "of interest" (Reliable Change Index between 1.64 and 1.95), a reliable change "of concern" (Reliable Change Index above 1.96), or an indeterminate/unchanged score (Reliable Change Index of between -1.96 and 1.63) (In review citation withheld for blind review). For a full description of the Reliable Change Index equation and statistic see Jacobson et al. (1984) and Christensen and Mendoza, (1986), for more information on determining the Reliable Change Index score see Wise (2004).

Using the Reliable Change Index described above, the NAID will be used to determine the presence or absence of cognitive deterioration over a period of 16 months in this study. Oliver amd Adams (2007) highlight that the NAID cannot be used to diagnose dementia, only identify cognitive deterioration. Whilst cognitive deterioration is essential for a diagnosis of dementia of the Alzheimer's type, without supporting reports of impact upon everyday living and a full medical screen, a full diagnosis of dementia cannot be made. It is for this reason that this paper will usethe term cognitive deterioration, and not dementia, for individuals who show changes "of interest" or "of concern" on at least two subscales of the NAID.

Executive Function: Four measures of executive function were taken from Ball et al. (2008) and administered at each assessment; the Tower of London (Krikorian, Bartok \& Gay, 1994), Weigl card sorting (Goldstein \& Scheerer, 1941), cats and dogs stroop task (Gerstadt, Hong \& Diamond, 1994) and scrambled boxes (Strauss \& Lewin, 1982).

Adaptive Behaviour: Informants who had known the individual for at least six months were interviewed using the Vineland Adaptive Behavior Scale - Survey Form (VABS; Sparrow et al., 1984). The original form of this interview was used as the $2^{\text {nd }}$ edition had not yet been published at the start of this study. 
Receptive language: The British Picture Vocabulary Scale $2^{\text {nd }}$ edition (BPVS; Dunn, Dunn, Whetton, \& Burley, 1998) mental age equivalents were used to determine the participant's ability for the purpose of ascertaining the degree of intellectual disability used in interpreting results on the NAID (see In review citation withheld for blind review).

Assessment for Adults with Developmental Disabilities. Described in full in (In review citation withheld for blind review), the Assessment for Adults with Developmental Disabilities (AADS; Kalsy et al., 2001) focuses solely upon describing an individual's behaviour and related consequences. This questionnaire comprises two sub-scales: behavioural excesses ( $\mathrm{n}=11$; e.g. restlessness, night and day wandering, vocally disruptive, taking items, crying, uncooperative, verbally and physically aggressive, sexually inappropriate and repetitive speech) and behavioural deficits ( $n=17$, e.g. inactivity, word finding difficulties, lack of interest, withdrawn, disoriented in time, person and place, becoming lost, two areas of self help, difficulties carrying out sequential acts, poor concentration, not alert, slow and falling) commonly associated with dementia. Informants were required to rate the frequency and the effect of the behaviours (on the carers (carer effect) and on the individual showing the behaviour (person effect)) on a Likert scale ranging from zero to six. In review (citation withheld for blind review) report the intra-class correlation coefficients for the frequency of excesses and deficits and the number of excesses and deficits as $.81, .80, .86$ and .80 respectively. For the management of excesses and deficits and the effect of excesses and deficits the intra-class correlation coefficients were $.76, .83, .59$ and .76 respectively.

\section{$\underline{\text { Data Analysis }}$}

\section{Determining cognitive deterioration}

This paper will focus upon comparing those with and without cognitive deterioration on measures of executive function, behaviour and adaptive behaviour. For the purpose of this paper, cognitive deterioration was deemed present if the participant achieved a Reliable Change Index score of at least 1.64 (i.e. a reliable decline "of interest" or "of concern") between baseline and sixteen month follow up on a minimum of two subscales of the NAID. This method of determining cognitive deterioration has good concurrent validity with both informant and independent neuropsychological measures of dementia (Oliver \& Adams, 2007; In review citation withheld for blind review). 
Prior to each analysis, measures of skew and kurtosis were generated and a Kolmogorov-Smirnov test undertaken to test for normality of the data. If assumptions concerning normality and homogeneity of the data could not be met then the data were transformed using logarithmic transformation (Howell, 1997). For all tests of difference, the independent variable was group (those with and those without cognitive deterioration) and the dependent variable was the result of the measure under test. SPSS 11.5 was used to carry out all statistical analysis.

\section{$\underline{\text { Results }}$}

Using the Reliable Change Index method described above, ten of the thirty individuals were classified as showing cognitive deterioration on at least two subscales of the NAID between baseline and sixteen month follow up. These participants differed from the remaining sample in terms of age (cognitive deterioration group mean $=48.9, s d=7.2$; no cognitive deterioration group mean=42.3, $s d=6.8 ; \mathrm{t}=-2.5, p=<.05)$ but did not differ with regard to gender, residential setting, vision, hearing or speech (based upon carer report); see Table 1. In order to ensure comparability in levels of ability between those with and without cognitive deterioration, mental age equivalents of the three subscales (communication, daily living skills, socialization) of the VABS were compared across the duration of the study in a $3 \times 3 \times 2$ (data collection point $x$ subscale $x$ group) analysis of variance (ANOVA). There were no significant interactions (communication: $\mathrm{F}(2,56)=.35, p>.05$; daily living skills $\mathrm{F}(2,56)=.76, p>.05$; socialisation $\mathrm{F}(2,56)=.66, p>.05)$ or main effects of time (communication: $\mathrm{F}(2,56)=1.2, \quad p>.05$; daily living skills $\mathrm{F}(2,56)=2.1, \quad p>.05 ;$ socialisation $\mathrm{F}(2,56)=1.4, p>.05$ ) or group (communication: $\mathrm{F}(2,56)=.57, p>.05$; daily living skills $\mathrm{F}(2,56)=1.6$, $p>.05$; socialisation $\mathrm{F}(2,56)=1.3, p>.05)$ suggesting that these two groups did not differ in terms of adaptive behaviour across the duration of the study.

++++insert Table 1 about here++++

The association between cognitive deterioration and performance on measures of executive function

In order to test the first hypothesis, that those showing cognitive deterioration will show impairments on measures of executive function in comparison to those without cognitive deterioration, a mean Z-score was derived across the four measures of executive functioning. This was done as per Schoenfeld et al. (1999) to minimise the number of comparisons made. For each of the four measures of executive function (tower of London, Weigl card sorting, cats and dogs and 
scrambled boxes), raw scores were transformed into Z-scores, the mean of which was calculated for each participant at baseline, 8 month and 16 month follow up. This mean Z-score was entered into a two-way, mixed design ANOVA (using the Greenhouse-Geisser correction method) as the dependent variable alongside the between subjects factor of presence or absence of cognitive deterioration and within-subjects factor of time point in the study. The results shown in Figure 1 revealed a significant interaction between the time point and group (presence or absence of cognitive deterioration $)(\mathrm{F}(1.79,50.2)=8.2, p<.001)$. There were also main effects of time $(\mathrm{F}(2,56)=3.66, p<.05)$ and group $(\mathrm{F}(1,28)=5.6, p<.05)$. Post hoc within group analyses (using repeated measures t-tests) revealed that those with cognitive deterioration show a significant decline on measures of executive function between baseline and sixteen month follow up $(\mathrm{t}(9)=3.9, p<.01)$ and eight and sixteen month follow up $(\mathrm{t}(9)=4.7, p<.001)$ but not between baseline and eight month follow up. Post hoc between group analyses (using independent t-tests) revealed a significant difference between mean total $\mathrm{z}$-scores at sixteen month follow up $(\mathrm{t}(28)=3.3, p<.01)$ but not between baseline and eight month follow up.

++++ Insert Figure 1 about here ++++

The association between cognitive deterioration and behavioural excesses and behavioural deficits on the AADS

Prior to analyses of the AADS, logarithmic transformations were undertaken on both the frequency scores and the raw (aggregate) and mean effect per behaviour scores of the effect subscales (carer effect, person effect) for behavioural excesses and behavioural deficits in order to minimise skew and kurtosis. After the logarithmic transformations, the transformed data met the assumptions of parametric statistics.

In order to test the second hypothesis, that those showing cognitive deterioration will evidence higher frequencies of behavioural excesses in comparison to those without cognitive deterioration, a two way mixed design ANOVA was conducted with the transformed behavioural excesses frequency score as the dependent variable, time point as the within-subject factors and group (presence or absence of cognitive deterioration) as the between-subjects factor. The resultsrevealed a significant interaction between time and group (presence or absence of cognitive deterioration); $\mathrm{F}(2,56)=6.5, p<.01$. The form of this interaction is represented in Figure 2. There were no main effects of time $(\mathrm{F}(2,56)=1.8, p=.18)$ or group $(\mathrm{F}(1,28)=.07, p=.79)$. Post-hoc comparisons revealed that it is between the eight and sixteen month follow up that there is a significant increase in 
frequencies of behavioural excesses, but only within those with cognitive deterioration $(\mathrm{t}(29)=-2.5$, $p<.05)$.

This analysis was then repeated with the transformed behavioural deficits frequency score as the dependent factor, in order to test the second hypothesis that informants of those showing cognitive deterioration will evidence higher frequencies of behavioural deficits in comparison to those without cognitive deterioration. The results revealed a significant interaction between the time score for behavioural deficits and group (presence or absence of cognitive deterioration); $\mathrm{F}(2$, $54.6)=5.2, p<.01$. The form of this interaction is represented in lower panel of Figure 2. Post-hoc comparisons revealed that it is not until sixteen month follow up that there is a significant difference in frequencies of behavioural deficits $(\mathrm{t}(29)=-3.4, p<.01)$. Significant main effects of group $(\mathrm{F}(1,28)=8.1 p<.01)$ and time $(\mathrm{F}(2,56)=4.4, p<.05)$ were also revealed which can be accounted for by the interaction (see Figure 2).

\section{++++ Insert Figure 2 about here ++++}

The final analyses focused upon the effect of behavioural excesses and behavioural deficits upon carers and upon the individual showing the behaviour. Due to differing numbers of items in the behavioural excesses $(n=11)$ and behavioural deficits $(n=17)$ subscales, the mean effect per behaviour score was calculated by dividing the total carer or person effect score by the number of behavioural excesses or behavioural deficits reported for that person. This transformation allowed for a direct comparison of the effect of behavioural excesses and behavioural deficits whilst minimising the number of analyses undertaken. Mean effect per behaviour scores, for carer and person effect of behavioural excesses and behavioural deficits were entered into a $3 \times 4 \times 2$ (time $x$ subscale $x$ group) mixed design ANOVA in order to directly compare the effect of behavioural excesses and behavioural deficits. There were no significant interactions, nor main effects of time. However, there was a significant main effect of group for both carer effect $(\mathrm{F}(1,28)=14.4, p<.001)$ and person effect $(\mathrm{F}(1,28)=14.9, p<.001)$. Inspection of the mean values across the three data collection points indicate that each behavioural excess or deficit shown by individuals with cognitive deterioration has a greater effect on carers (mean=.77, $s d=1.1$ ) and the individual displaying the behaviour (mean=.80, $s d=1.1$ ) than those shown by those without cognitive deterioration (mean carer $=.3, s d=.51$, mean person=.17, $s d=.38$ ).

Examining the relationship between changes in performance on tasks of executive function and changes in behavioural excesss and behavioural deficits on the AADS 
In order to examine the relationship between decline on measures of executive function and change in the frequency of behavioural excesses and the frequency of behavioural deficits, Pearson's correlations analyses were undertaken for the total sample and for those with cognitive deterioration. Partial correlations (using the "early" signs domain score) were then undertaken in order to control for the effects of deterioration in memory

For ease of interpretation, changes are presented so that all negative scores represent decline (i.e. an increase in frequency of behavioural excesses or behavioural deficits, or a decline in performance on measures of executive function). For all measures, change was investigated between each data collection point.

++++insert Table 2 about here++++

Table 2 shows that changes on measures of executive function are only significantly correlated with changes in behavioural excesses in those with cognitive deterioration. Behavioural deficits show very little association with changes in executive function in either sample. The partial correlations indicate that these relationships between change in behavioural excesses and decline on measures of executive function in those with cognitive deterioration is not simply due to a decline in memory. However, it is important to note the small sample sizes for such correlations.

\section{Discussion}

In this study we used an empirical method for determining decline on an established neuropsychological assessment to examine changes on measures of executive function and behaviour in adults with Down syndrome with and without early cognitive deterioration. The results showed that individuals with Down syndrome with early cognitive deterioration demonstrated a significant decrease on measures of executive function alongside a significant increase in frequency of behavioural excesses and behavioural deficits across a sixteen month period, a pattern not shown by those without cognitive deterioration. Carers of individuals with cognitive deterioration reported significantly greater carer and person effects of behavioural excesses and behavioural deficits than carers of individuals without cognitive deterioration. Finally, correlational analyses suggest that a decline in performance on measures of executive function is only associated with an increase in behavioural excesses in those with cognitive deterioration and that this association is not associated with the decline in memory. There were no significant differences or declines in adaptive behaviour when assessed using the VABS in those with or 
without cognitive deterioration, suggesting that the declines seen are not simply the result of global deterioration, but caused by early, specific change.

This study has highlighted the importance of considering a broad spectrum of assessment in the early stages of dementia in individuals with Down syndrome to reflect the potentially multiple underlying neuropathological causes and stages. Although only one measure of cognitive deterioration was used, the statistically based method was based upon longitudinal change on tests of memory, language and praxis. All of these areas are strongly related to temporal and parietal lobes of the brain. The significant decline on measures of executive function only in those with cognitive deterioration suggests that the frontal lobes of those showing cognitive deterioration are also affected by underlying neuropathology. While the association between tests of executive function and the frontal lobes is not simple (Miyake et al., 2000), tests of executive function provide accurate and valid estimates of frontal lobe functioning (see Sergeant, Geurts \& Oosterlaan, 2002, for a review).

This finding extends the current discussion surrounding the reserve capacity hypothesis (Holland $e t$ al., 1998; 2000; Mortimer, 1988) as a possible explanation for differences in the clinical presentation of dementia in individuals with Down syndrome. This hypothesis suggests that, due to compromised frontal lobes from birth, individuals with Down syndrome have a low frontal lobe "reserve capacity" making this area susceptible to early neuropathological changes. This hypothesis would therefore suggest that evidence of frontal lobe deterioration should be evident in individuals showing cognitive deterioration. The results of this study provide some support for this suggestion; individuals showing deterioration in areas of cognition related to the temporal and parietal lobes of the brain evidenced change on measures of executive function that was not apparent in individuals with no evidence of cognitive deterioration. However, the exact course of deterioration (i.e. declines in temporal and parietal function happened before or after declines in the frontal lobes) cannot be established from this small data set. Graphically, Figure 1 shows that those with cognitive deterioration are performing worse (but not at a statistically significantl level) than those without cognitive deterioration on measures of executive function at baseline. The reserve capacity hypothesis would suggest that the frontal lobes of individuals with cognitive deterioration were compromised pre-baseline by Alzheimer's related neuropathology. Alternative explanations must be also considered, including the possibility of the frontal lobes of individuals with cognitive deterioration being compromised pre-baseline through underlying neurological deficits (i.e. smaller frontal lobes from birth). Despite multiple explanations for lower baseline performance of individuals with cognitive deterioration on measures of executive function, Holland et al.'s reserve 
capacity hypothesis provides a strong rationale for the cause of the longitudinal decline. Overall, these results provide support for extending the use of tests of executive function in longitudinal studies of dementia in adults with Down syndrome.

Using a longitudinal design, it was possible to identify that changes in behaviour emerged alongside cognitive decline, as reported within the general population. However, what is not known from these data is whether the declines identified on the NAID were at a level that would present clinically and consequently be identified by carers as declines or difficulties in day-to-day cognitive functioning. It is therefore important that carers and health professionals are vigilant for behavioural changes as potential early clinical indicators of cognitive or neuropathological changes and/or dementia. Carers are more likely to seek help and referral on the basis of behavioural excesses than behavioural deficits, which may be due to their perceived impact upon care giving and the wider environment (Adams et al., 2008). However, as this study has highlighted that both behavioural excesses and behavioural deficits are present in those showing cognitive deterioration, it is important that both are considered in any assessment for dementia.

This study is the first to show associations between changes in behaviour and changes on measures of executive function in individuals with Down syndrome showing cognitive deterioration. Associations were identified between an increased frequency of behavioural excesses and declines on measures of executive function only in those with cognitive deterioration, which were not solely attributable to declines in memory. However, associations were not identified between changes in frequency of behavioural deficits and declines on measures of executive function. The differing patterns of frequency of behavioural excesses and behavioural deficits may reflect different underlying neuropathological stages or causes. Behavioural excesses are strongly related to the functions of the frontal lobes whereas behavioural deficits have been related to various regions of neuropathology (Bozeat, Gregory, Lambon, Ralph \& Hodges, 2000). It may be hypothesised that the initial increase in behavioural deficits may reflect early neuropathological change in the temporal and parietal lobes (causing symptoms such as forgetting words and belongings, reduced ability to follow instructions). The increase between eight and sixteen month follow ups may be caused by neuropathology in the frontal lobes exceeding the threshold for clinical presentation, causing either an increased frequency or quantity of frontal related behavioural deficits (e.g. apathy, disinterest). Future research could longitudinally investigate the AADS at item levelin order to gather further information on the exact pattern of change within the catergories of behavioural excesses and behavioural deficits and provide support for or against this hypothesis. 
These results also provide strong evidence for prospective screening strategies for dementia in adults with Down syndrome which encompass neuropsychological and informant based assessments of dementia. Adams and Oliver (2007) provided single point and longitudinal score interpretation methods and this study has demonstrated that they may be more sensitive to cognitive deterioration than informant based assessments of dementia. Clinically, identifying links between impairment in a particular area of cognitive function and changes in behavioural presentation may have important implications for management and care issues. This study shows that, in the stages prior to a diagnosis of dementia, adults with Down syndrome show an increased frequency of behavioural excesses and deficits. Importantly, informants are reporting that some of these behaviours have a significant impact upon carers and upon the person displaying the behaviour. Early detection and intervention is undeniably more effective in this and other populations (see Simeonsson, Cooper \& Scheiner, 1982; Smith, 1999) due to a lower frequency and intensity of the problem behaviours. However, it is not until behavioural changes such as those noted within this study pose a significant management problem that carers of individuals with dementia seek specialist help both for people with Down syndrome (Adams et al., 2008) and in the general population (Duggan, Lewis \& Morgan, 1996). It is therefore imperative that individuals with Down syndrome are screened prospectively, and such screening programmes draw upon the literature (such as those reported within this paper) to identify the earliest signs of decline or deterioration that may be indicative of the early signs of dementia.

It is important to recognise the methodological limitations of this study. The sample size, especially those with cognitive deterioration, is very small. The external validity of this study may have been compromised through this and the number of individuals excluded for the latter stages of the analysis through floor effects. It must also be remembered that only one measure of decline was used, and whilst Oliver and Adams (2007) have shown excellent concurrent validity with carer based reports on the CAMDEX-DS, this method of identifying decline in cognitive performance is still very much in its infancy. While the relationship between measures of executive function and the frontal lobes is now accepted throughout the literature, assuming this direct link may affect the construct validity of the study. Administering measures of executive function at three time points may effect the internal validity of the study as, by definition, measures of executive function should not be repeated. However, such methods have been used and accepted within the wider literature (e.g. Griffith, Pennington, Wehner \& Rogers, 1999) and may be the only way to map the course of decline within this area.

\section{Acknowledgements}


The authors would like to thank the individuals with Down syndrome, their families and carers alongside Dr Sunny Kalsy, Simone Peters and Murielle Broquard for their continued support throughout the duration of this project. 


\section{References}

Adams, D., Oliver, C., Kalsy, S., Peters, S., Broquard, M., Basra, T. et al. (2008).

Behavioural characteristics associated with dementia assessment referrals in adults with Down syndrome. J.Intellect.Disabil.Res., 52, 358-368.

Baddeley, A. (1998). The central executive: a concept and some misconceptions. $J$ Int.Neuropsychol.Soc., 4, 523-526.

Ball, S. L., Holland, A. J., Treppner, P., Watson, P. C., \& Huppert, F. A. (2008). Executive dysfunction and its association with personality and behaviour changes in the development of Alzheimer's disease in adults with Down syndrome and mild to moderate learning disabilities. Br.J.Clin.Psychol., 47, 1-29.

Blair, M., Marczinski, C.A., Davis-Faroque, N., \& Kertesz, A. (2007). A longitudinal study of language decline in Alzheimer's disease and frontotemporal dementia. Journal of the International Neuropsychological Society, 13, 237-245

Bozeat, S., Gregory, C. A., Ralph, M. A., \& Hodges, J. R. (2000). Which neuropsychiatric and behavioural features distinguish frontal and temporal variants of frontotemporal dementia from Alzheimer's disease? J Neurol.Neurosurg.Psychiatry, 69, 178-186.

Christensen, L. \& Mendoza, J. L. (1986). A method of assessing change in a single subject: An alteration of the RC Index. Behavior Therapy, 17, 305-308.

Crayton, L., Oliver, C., Holland, A. J., Bradbury, J., \& Hall, S. (1998). The neuropsychological assessment of age related cognitive deficits in adults with Down's syndrome. Journal of Applied Research in Intellectual Disabilties, 11, 255-272.

Devenny, D. A., Zimmerli, E. J., Kittler, P., \& Krinsky-McHale, S. J. (2002). Cued recall in early-stage dementia in adults with Down's syndrome. J.Intellect.Disabil.Res., 46, 472-483. 
Duggan, L., Lewis, M., \& Morgan, J. (1996). Behavioural changes in people with learning disability and dementia: a descriptive study. J.Intellect.Disabil.Res., 40 ( Pt 4), 311-321.

Dunn, L. M., Dunn, L. M., Whetton, C., \& Burley, J. (1998). British Picture Vocabulary Scales (Version 2). Windsor, NFER-Nelson.

Gerstadt, C. L., Hong, Y. J., \& Diamond, A. (1994). The relationship between cognition and action: performance of children 3 1/2-7 years old on a Stroop-like day-night test. Cognition, 53, 129-153.

Goldstein, K. \& Scheerer, M. (1941). Abstract and concrete behaviour; an experimental study with special tests. Psychological Monographs, 151.

Griffith, E. M., Pennington, B. F., Wehner, E. A., \& Rogers, S. J. (1999). Executive functions in young children with autism. Child Dev., 70, 817-832.

Holland, A. J., Hon, J., Huppert, F. A., Stevens, F., \& Watson, P. (1998). Population-based study of the prevalence and presentation of dementia in adults with Down's syndrome. Br.J.Psychiatry, 172, 493-498.

Holland, A. J., Hon, J., Huppert, F. A., \& Stevens, F. (2000). Incidence and course of dementia in people with Down's syndrome: findings from a population-based study. J.Intellect.Disabil.Res., 44 ( Pt 2), 138-146.

Howell, D. C. (1997). Statistical Methods for Psychology. Belmont, CA: Duxbury Press.

Jacobson, N. S., Follette, W. C. \& Revenstorf, D. (1984). Psychotherapy outcome research: methods for reporting variability and evaluating clinical significance. Behavior Therapy, 15, 336352 
Kalsy S., McQuillan S., Oliver C. \& Hall S. (2001) Assessment for Adults with Developmental Disabilities. University of Birmingham, Birmingham.

Krikorian, R., Bartok, J., \& Gay, N. (1994). Tower of London procedure: a standard method and developmental data. J Clin.Exp.Neuropsychol., 16, 840-850.

Miyake, A., Friedman, N., Emerson, M., Witzki, A., Howerter, A., \& Wager, T. (2000). The Unity and Diversity of Executive Functions and Their Contributions to Complex "Frontal Lobe" Tasks: A Latent Variable Analysis. Cognitive Psychology, 41, 49-100.

Mortimer, J. A. (1988). Do psychosocial risk factors contribute to Alzheimer's disease? In A.Henderson \& J. Hendersen (Eds.), Etiology of Dementia of Alzheimer's Type (pp. 39-52). John Wiley \& Sons Ltd..

Nelson, L., Lott, I., Touchette, P., Satz, P., \& D'Elia, L. (1995). Detection of Alzheimer disease in individuals with Down syndrome. Am.J.Ment.Retard., 99, 616-622.

Nelson, L., Orme, D., Osann, K., \& Lott, I. T. (2001). Neurological changes and emotional functioning in adults with Down Syndrome. J.Intellect.Disabil.Res., 45, 450-456.

Oliver, C. \& Adams,D. (2007). Manual for score interpretation of the Neuropsychological Assessment of Dementia in Individuals with Intellectual Disabilities. Unpublished: University of Birmingham.

Oliver, C. \& Crayton, L. (1993). Neuropsychological Assessment of Dementia in Individuals with Intellectual Disabilities. Unpublished: University of Birmingham.

Oliver, C., Crayton, L., Holland, A., Hall, S., \& Bradbury, J. (1998). A four year prospective study of age-related cognitive change in adults with Down's syndrome. Psychol.Med., 28, 13651377. 
Oliver, C., Holland, T., Hall, S., \& Crayton, L. (2005). Effects of increasing task load on memory impairment in adults with Down syndrome. Am.J.Ment.Retard., 110, 339-345.

Pinter, J. D., Eliez, S., Schmitt, J. E., Capone, G. T., \& Reiss, A. L. (2001). Neuroanatomy of Down's syndrome: a high-resolution MRI study. Am.J.Psychiatry, 158, 1659-1665.

Prasher, V. P. \& Filer, A. (1995). Behavioural disturbance in people with Down's syndrome and dementia. J.Intellect.Disabil.Res., 39 ( Pt 5), 432-436.

Prasher, V., Cumella, S., Natarajan, K., Rolfe, E., Shah, S., \& Haque, M. S. (2003). Magnetic resonance imaging, Down's syndrome and Alzheimer's disease: research and clinical implications. J.Intellect.Disabil.Res., 47, 90-100.

Schoenfeld, J., Seidenberg, M., Woodard, A., Hecox, K., Inglese, C., Mack, K. et al. (1999). Neuropsychological and behavioral status of children with complex partial seizures. Dev.Med.Child Neurol., 41, 724-731.

Sergeant, J. A., Geurts, H., \& Oosterlaan, J. (2002). How specific is a deficit of executive functioning for attention-deficit/hyperactivity disorder? Behav.Brain Res., 130, 3-28.

Simeonsson, R. J., Cooper, D. H., \& Scheiner, A. P. (1982). A review and analysis of the effectiveness of early intervention programs. Pediatrics, 69, 635-641.

Smith, T. (1999). Outcome of Early Intervention for Children With Autism. Clinical Psychology: Science and Practice, 4, 33-35.

Sparrow, S. S., Balla, D. A., \& Chiccetti, D. V. (1984). Vineland Adaptive Behavior Scales: Interview Edition, Survey Form Manual. Circle Pines, MN, American Guidance Service. 
Strauss, H. \& Lewin, I. (1982). Am empirical study of the Weigl-Goldstein-Scheerer ColorForm Test according to a developmental frame of reference. Journal of clinical Psychology, 38, 367-375.

Stuss, D. T. \& Benson, D. F. (1984). Neuropsychological studies of the frontal lobes. Psychol.Bull., 95, 3-28.

Urv, T. K., Zigman, W. B., \& Silverman, W. (2003). Maladaptive behaviors related to adaptive decline in aging adults with mental retardation. Am.J.Ment.Retard., 108, 327-339.

Wise, E. A. (2004). Methods for analyzing psychotherapy outcomes: a review of clinical significance, reliable change, and recommendations for future directions. J.Pers.Assess., 82, 50-59. 
No Cognitive Deterioration $-\mathrm{O}=$ Cognitive Deterioration

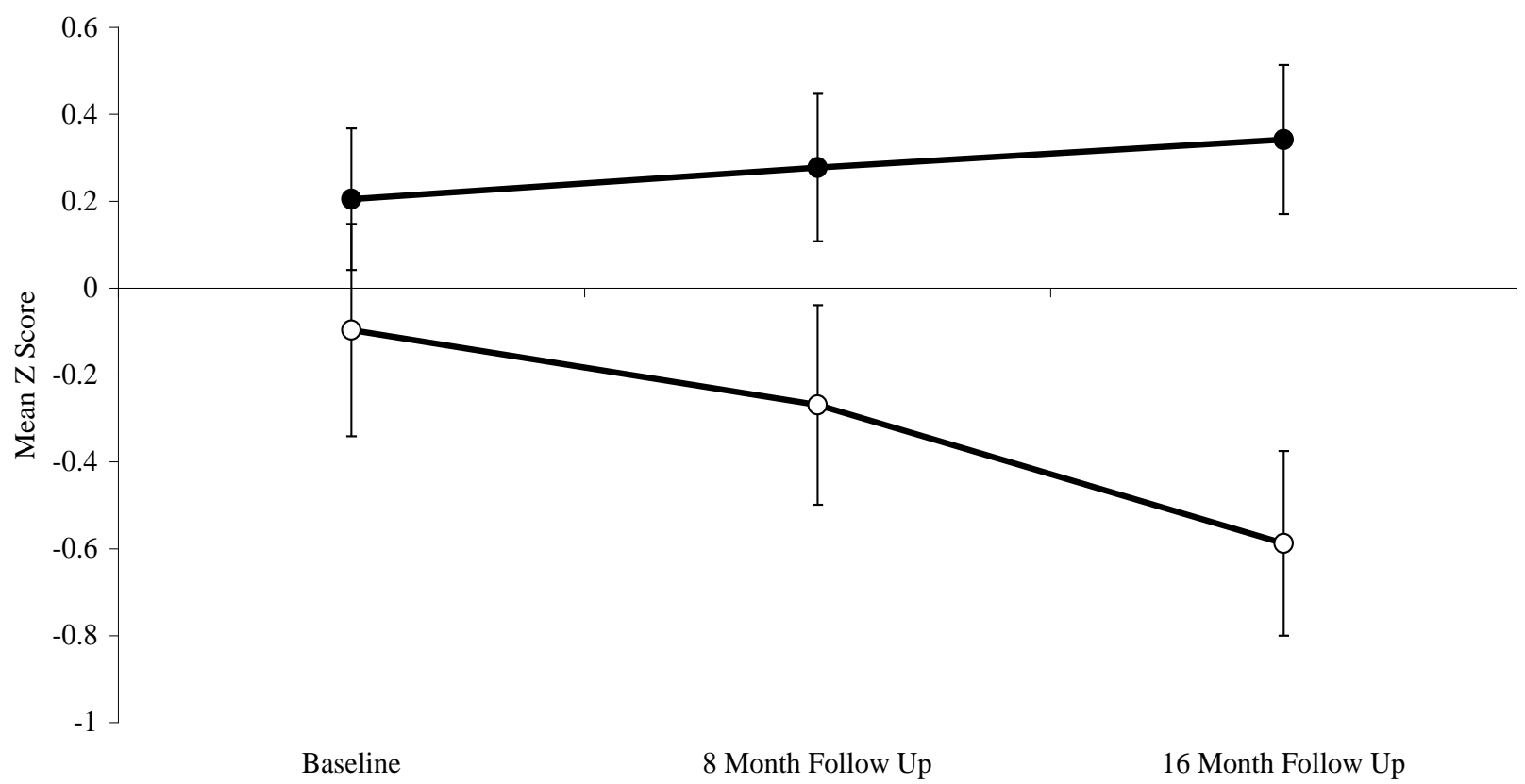

Figure 1: Mean total Z-Scores ( \pm 1 standard error) on measures of executive functioning for those with and without cognitive deterioration 


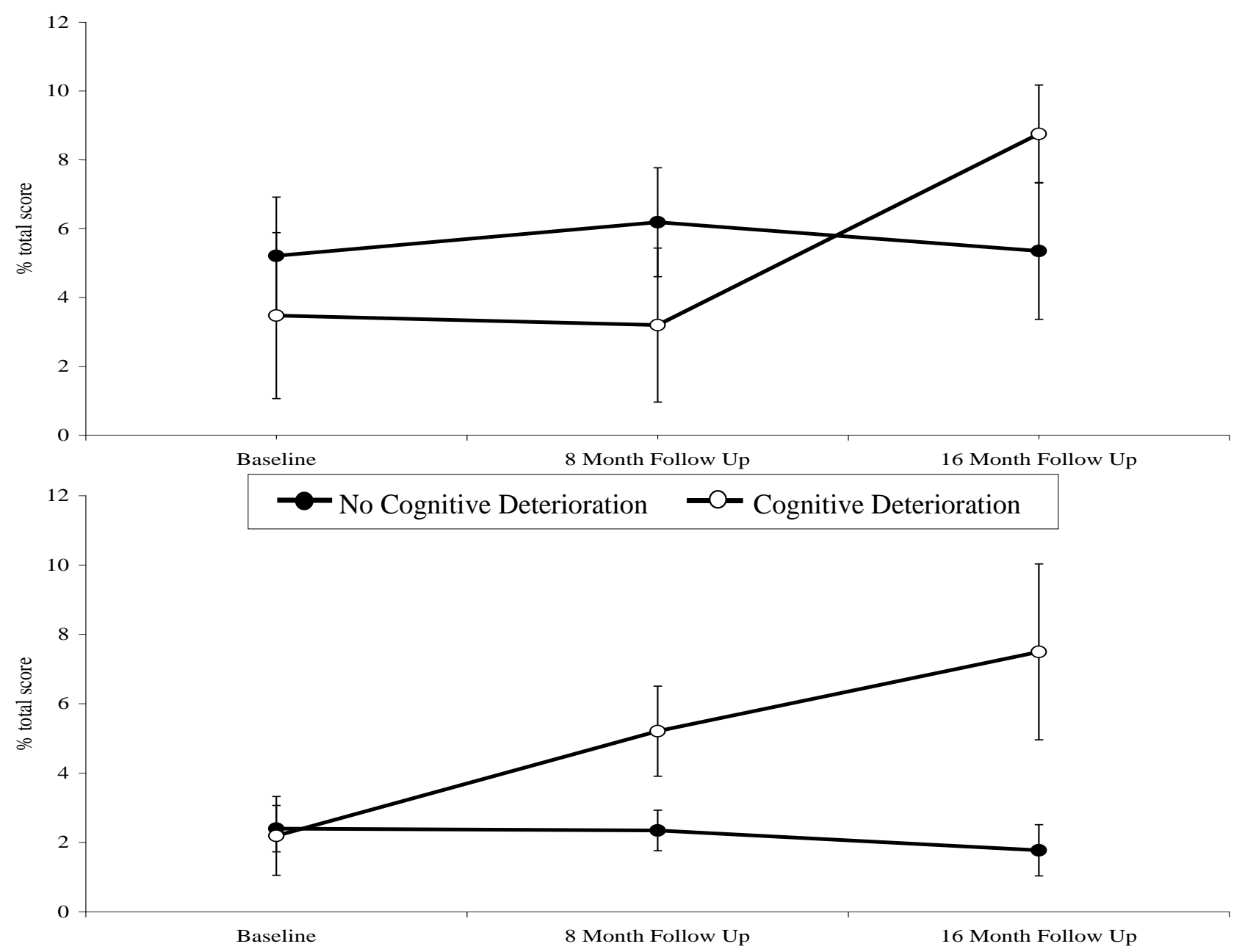

Figure 2: Total frequency score for behavioural excesses (upper panel) and behavioural deficits (lower panel) ( \pm 1 standard error) for those with and without cognitive deterioration 
Table 1: Comparison of demographic characteristics and adaptive behaviour (MA months) of those with and without cognitive deterioration

\begin{tabular}{|c|c|c|c|c|c|c|}
\hline $\mathrm{N}$ & & $\begin{array}{c}\text { Cognitive } \\
\text { Deterioration } \\
10\end{array}$ & $\begin{array}{c}\text { No Cognitive } \\
\text { Deterioration } \\
20\end{array}$ & $\mathrm{t} / \chi^{2}$ & df & $\mathrm{p}$ \\
\hline Gender & Male & $\begin{array}{c}5 \\
(50 \%)\end{array}$ & $\begin{array}{c}10 \\
(50 \%)\end{array}$ & 0 & 1 & 1 \\
\hline Age & $\begin{array}{l}\text { Mean } \\
s d\end{array}$ & $\begin{array}{l}48.9 \\
(7.2)\end{array}$ & $\begin{array}{l}42.3 \\
(6.8)\end{array}$ & -2.5 & 28 & .02 \\
\hline Accommodation & $\begin{array}{l}\text { Family Home } \\
\text { Residential Home }\end{array}$ & $\begin{array}{c}3 \\
(30 \%) \\
7 \\
(70 \%)\end{array}$ & $\begin{array}{c}6 \\
(30 \%) \\
14 \\
(70 \%)\end{array}$ & 0 & 1 & 1 \\
\hline Day placement & $\begin{array}{l}\text { Yes } \\
\text { No }\end{array}$ & $\begin{array}{c}10 \\
(100 \%) \\
0 \\
(0 \%)\end{array}$ & $\begin{array}{c}19 \\
(95 \%) \\
1 \\
(5 \%)\end{array}$ & .517 & 1 & .67 \\
\hline Hypothyroidism & $\begin{array}{l}\text { Yes } \\
\text { No }\end{array}$ & $\begin{array}{c}4 \\
(40 \%) \\
6 \\
(60 \%)\end{array}$ & $\begin{array}{c}5 \\
(25 \%) \\
15 \\
(75 \%)\end{array}$ & .714 & 1 & .43 \\
\hline Vision & $\begin{array}{l}\text { Normal/Corrected } \\
\text { Poor }\end{array}$ & $\begin{array}{c}8 \\
(80 \%) \\
2 \\
(20 \%)\end{array}$ & $\begin{array}{c}20 \\
(100 \%) \\
0 \\
(0 \%)\end{array}$ & 4.3 & 1 & .10 \\
\hline Hearing & $\begin{array}{l}\text { Normal/Corrected } \\
\text { Poor }\end{array}$ & $\begin{array}{c}9 \\
(90 \%) \\
1 \\
(10 \%)\end{array}$ & $\begin{array}{c}16 \\
(80 \%) \\
4 \\
(20 \%)\end{array}$ & .48 & 1 & .45 \\
\hline & Sentences & $\begin{array}{c}9 \\
(90 \%)\end{array}$ & $\begin{array}{c}16 \\
(80 \%)\end{array}$ & & & \\
\hline Speech & Odd words & $\begin{array}{c}1 \\
(10 \%)\end{array}$ & $\begin{array}{c}4 \\
(20 \%)\end{array}$ & .94 & 1 & .33 \\
\hline \multicolumn{2}{|l|}{$\underline{\text { Adaptive Behaviour }}$} & $\begin{array}{l}\text { Mean } \\
(s d)\end{array}$ & $\begin{array}{l}\text { Mean } \\
(s d)\end{array}$ & $\mathrm{F}$ & $\mathrm{df}$ & $\mathrm{p}$ \\
\hline Vineland & Communication & $\begin{array}{c}43.2 \\
(17.3)\end{array}$ & $\begin{array}{c}50.9 \\
(28.2)\end{array}$ & .57 & 2,56 & .43 \\
\hline Behaviour Scales & $\begin{array}{l}\text { Daily Living } \\
\text { Skills }\end{array}$ & $\begin{array}{c}77.6 \\
(28.9)\end{array}$ & $\begin{array}{c}87.3 \\
(46.3)\end{array}$ & 1.6 & 2,56 & .22 \\
\hline (MA months) & Socialisation & $\begin{array}{c}63.9 \\
(40.2)\end{array}$ & $\begin{array}{c}83.1 \\
(47.9)\end{array}$ & 1.3 & 2.56 & .52 \\
\hline
\end{tabular}


Table 2: Pearson's correlations and partial correlations between change in measures of executive function and change in behavioural excesses and deficits on the AADS for the total sample and those with cognitive deterioration

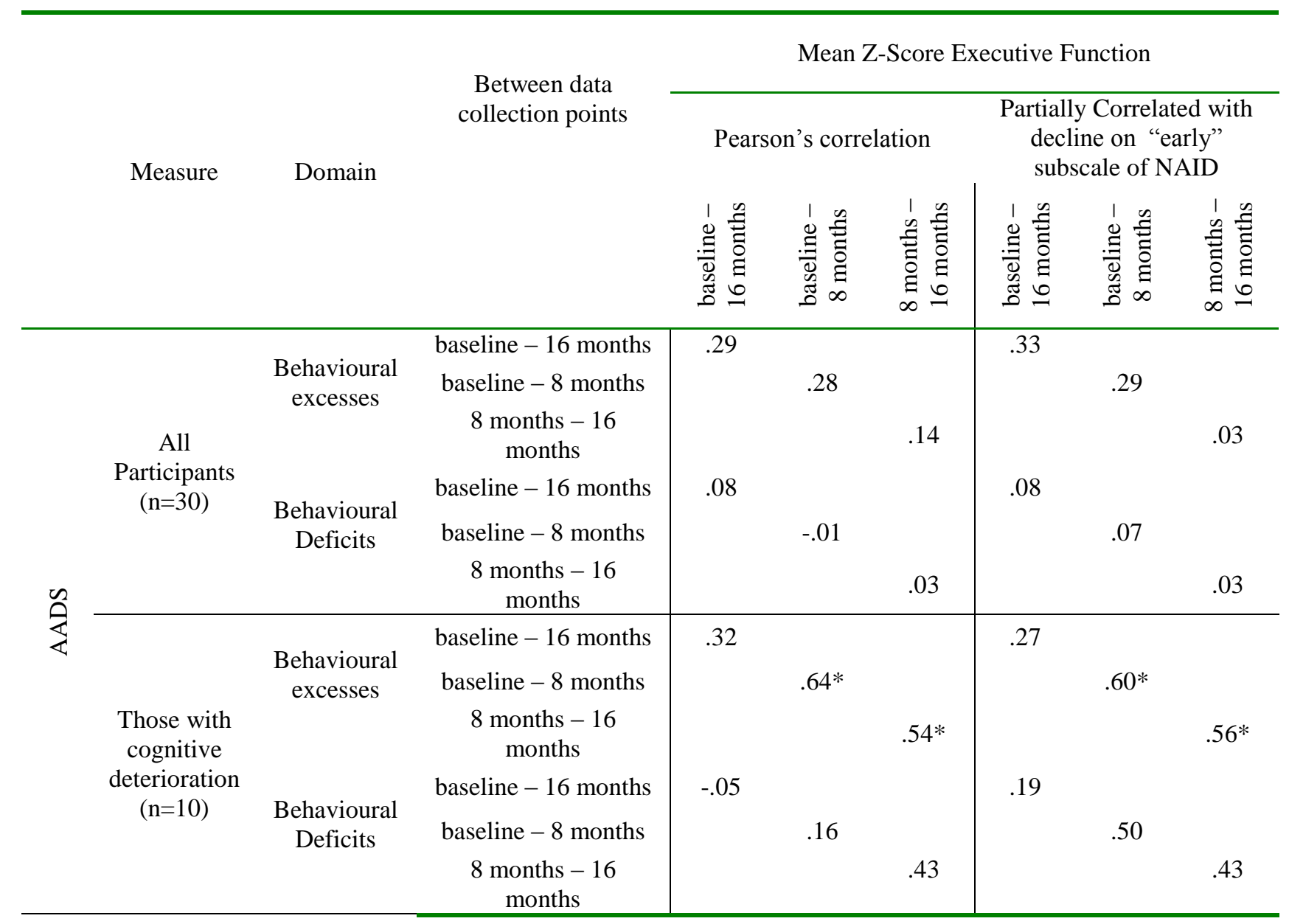

*: significant at $p=.01$ 